\title{
Study of a Piecewise Linear Dynamic System with Negative and Positive Stiffness
}

\author{
Keguan Zou ${ }^{\mathrm{a}}$, Satish Nagarajaiah ${ }^{\mathrm{a}, \mathrm{b}, *}$ \\ ${ }^{a}$ Department of Civil and Environmental Engineering, Rice University, Houston, Texas 77005, United States \\ ${ }^{b}$ Department of Mechanical Engineering, Rice University, Houston, Texas 77005, United States
}

\begin{abstract}
The present paper mainly focuses on numerical and analytical study of a piecewise linear dynamic oscillator with negative stiffness followed by positive stiffness which has not been studied to date. The dynamic system of interest stems from a previous analytical and experimental research on adaptive negative stiffness for the purpose of seismic protection. Numerical algorithms meant specifically for simulating piecewise smooth (PWS) systems like this nonlinear system are studied. An appropriate combination of negative stiffness and adequate damping can reduce the peak restoring or transmitted force with a slightly larger peak displacement. Essentially, the negative stiffness system in a dynamic system is very beneficial in reducing the amount of force transmitted. The exact solution is derived for free vibration. A modified Lindstedt-Poincaré method (modified L-P method) is adopted to derive approximate periodic solutions for the forced and damped system and its frequency-response curves are obtained through numerical simulation. The modified L-P solution obtained for the forced and damped case is found to agree well with the numerical results. In the piecewise linear dynamic system with initial negative stiffness followed by positive stiffness, it is found that the response remains bounded in a limit cycle. This system behaves similar to a van der Pol oscillator wherein negative damping is followed by positive damping. Presented herein is a special case as defined by the specified parameter ranges; thus, to make it more general future work is needed.
\end{abstract}

Keywords: Piecewise smooth system; negative stiffness; modified Lindstedt-Poincaré method; analytical approximation

\section{Introduction}

When investigating nonlinear systems, piecewise smooth (PWS) systems are common. Among them, piecewise linear systems are important since a large number of nonlinear systems can be approximated by using piecewise linear systems. Shaw and Holmes analyzed the harmonic, subharmonic and chaotic motions of a single-degree-of-freedom oscillator with a bilinear restoring force [1]. The method of a Poincaré section and the resultant return map were used to investigate the periodic orbits and local bifurcations. Wiercigroch and Sin studied the system dynamics of a base-excited piecewise linear oscillator with all positive stiffnesses [2]. Experimental and numerical results were arranged into bifurcation diagrams and Lissajous curves, compared, and analyzed. Luo proposed a local theory for non-smooth dynamic system to analyze the local singularity and transversality on the connecting boundary between adjacent domains [3]. Luo developed a methodology based on a perturbation method to investigate the local singularity of a periodically forced, piecewise linear system $[4,5]$. The mathematical criteria for detecting the grazing bifurcation was derived and numerical examples were given. The fragmentation of the strange attractors in this piecewise linear dynamic

\footnotetext{
${ }^{*}$ Corresponding author at: Department of Civil and Environmental Engineering, Rice University, Houston, Texas 77005, United States. Tel.: +1 7133486207.

Email address: satish.nagarajaiah@rice.edu (Satish Nagarajaiah)
} 
system was investigated mathematically and numerically. Vestroni et al. investigated a two-degree-offreedom piecewise linear system through the standard L-P method, which is valid for nonlinear systems with weak nonlinearity [6]. Pilipchuk combined nonsmooth temporal transformation and a standard perturbation procedure in order to derive closed-form asymptotic solutions for undamped, unforced, piecewise linear single- and two-degree-of-freedom oscillators where the stiffness jump was small [7].

Numerical simulation of piecewise smooth dynamic systems is a well-developed technique [8]. Several algorithms for simulating piecewise smooth dynamic systems have been available since the 1990's. The eventdriven algorithm [9] and algorithms for solving linear complementarity problems (LCP) [10] are widely used. These algorithms apply to Filippov differential systems [11], which include nonsmooth differential systems with discontinuities either in the states or their derivatives.
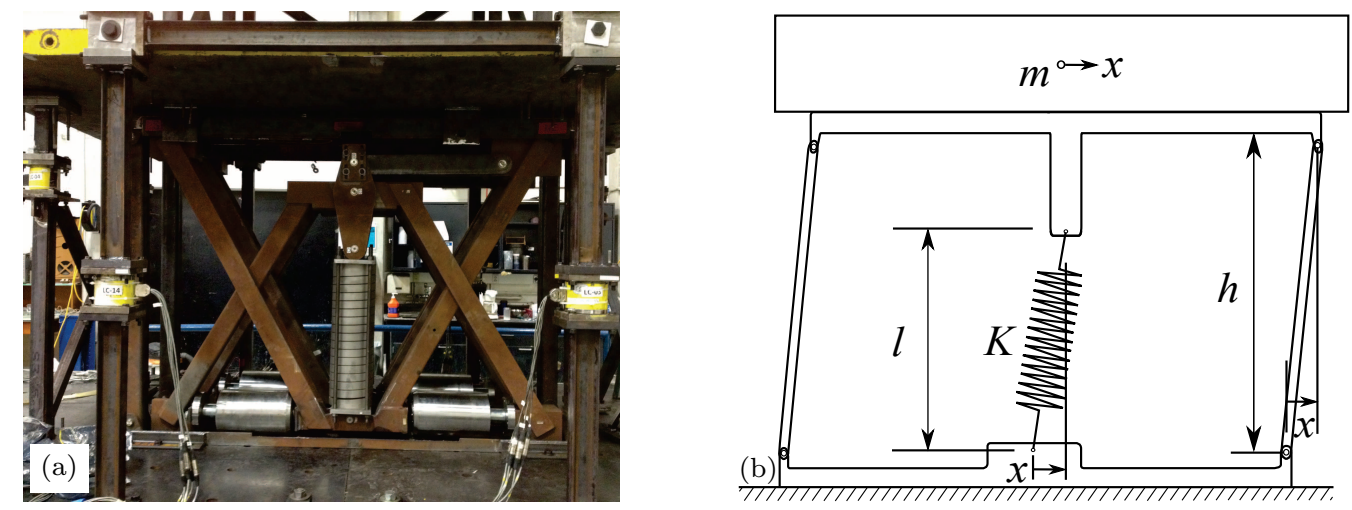

Fig. 1. Negative Stiffness Device (NSD). (a) Real negative stiffness device in experiment; (b) theoretical model.

The dynamic system investigated in the present work is motivated by a previous analytical and experimental study on the effect of adaptive negative stiffness in seismic protection [12-15]. The negative stiffness device (NSD) used in the experiment is shown in Fig. 1(a). The experimental force-displacement loops are presented in Pasala et al. [13] and Sarlis et al. [14]. A theoretical model of the negative stiffness device can be found in Nagarajaiah and Reinhorn [16], and is shown in Fig. 1(b). This theoretical model presents the principle of a negative stiffness device, which contains a precompressed spring with stiffness $K$. Whenever the mass, which is represented by the frame and mass in Fig. 1(b), moves left or right, the precompressed spring will further push it in the same direction as the displacement, and this yields an interesting negative stiffness behavior. The force-displacement relationship of the theoretical model is shown as the dashed line in Fig. 2, wherein it's compared to an idealized force-displacement relationship about which more details will be provided in the next section.

The theoretical restoring force (dashed line in Fig. 2) assumes the form of

$$
f_{1}(x)=K\left(1-\frac{l+\underline{\Delta}}{\sqrt{l^{2}+x^{2}}}\right) x
$$

where $\Delta$ is the precompressed length of the spring in Fig. 1(b). The restoring force shown in Fig. 2 is prepared with $K=0.4, \underline{\Delta}=1.0$, and $l=0.8$.

It has been verified by experiments that using adaptive negative stiffness as a supplemental device can significantly reduce the response, which poses the need for an analytical investigation of its dynamic characteristics. The dynamic system with negative stiffness is idealized as piecewise linear in the present work and the comparison of the idealized force-displacement with the theoretical force-displacement loop is shown in Fig. 2. Carrella et. al. studied the force transmissibility of a passive isolator with quasi-zero stiffness [17]. The quasi-zero stiffness isolator consisted of three linear springs including two pre-stressed oblique springs and one vertical spring. The restoring force produced by these three springs could be 


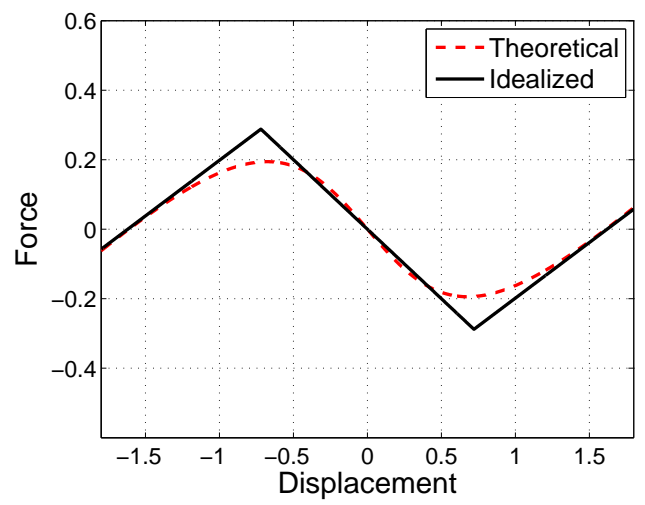

Fig. 2. Comparison of force-displacement relationships.

approximated as a purely cubic function of the displacement, but without the negative stiffness segment in the restoring force that is under consideration in this study, as shown in Fig. 2.

Nonlinear ordinary differential equations (ODEs) arise ubiquitously throughout science, engineering, natural phenomena and human activities. In contrast to linear ODEs, which can be easily solved analytically, most types of nonlinear ODEs cannot be solved analytically for the time being. In the past decades, powerful computational tools for the numerical simulation of nonlinear differential equations have become available. It is, however, sometimes preferable if analytical solutions can be obtained in special cases, which would provide a better understanding of the dynamic characteristics. Perturbation methods [18-20] are very popular for acquiring periodic solutions of nonlinear differential equations. A number of attempts have been made to seek analytical methods applicable to nonlinear differential equations with large parameters. Burton put forward a modified Lindstedt-Poincaré method which can be applied to strongly nonlinear dynamic systems [21]. Burton and Rahman further adapted the method of multiple scale to address strongly nonlinear oscillators [22]. Cheung et al. proposed a new parameter expansion technique which could keep the expansion parameter always small regardless of the magnitude of the original system parameters, and hence the standard Lindstedt-Poincaré method (L-P method) would be applicable thereafter [23].

In the present work, a piecewise linear dynamic system with two discontinuous points, negative stiffness, damping, and excitation is first numerically simulated, and then its analytical approximation is obtained in special cases and compared with the simulation results. The frequency-response curves and limit cycles from both methods are then compared. Time histories are furnished to further verify the frequency-response curves and gain more insights into the dynamics of the piecewise negative stiffness oscillator. The analysis presented in this work is a special case as defined by the specified parameter ranges; thus, to make it more general future work is needed.

\section{Problem Statement}

The dynamic system with piecewise linear spring force is as follows:

$$
m \frac{\mathrm{d}^{2} \hat{x}}{\mathrm{~d} \hat{t}^{2}}+c \frac{\mathrm{d} \hat{x}}{\mathrm{~d} \hat{t}}+f_{s}(\hat{x})=\hat{F} \cos \hat{\Omega} \hat{t},
$$

where the spring force

$$
f_{s}(\hat{x})=k_{1} \hat{x}+\Delta k[(\hat{x}-d) H(\hat{x}-d)+(\hat{x}+d) H(-\hat{x}-d)]=\left\{\begin{array}{lc}
k_{2} \hat{x}+\left(k_{2}-k_{1}\right) d & \hat{x}<-d \\
k_{1} \hat{x} & -d \leq \hat{x} \leq d \\
k_{2} \hat{x}-\left(k_{2}-k_{1}\right) d & \hat{x}>d
\end{array}\right.
$$

where $k_{1}<0, k_{2}=k_{1}+\Delta k>0, H(\cdot)$ is the heaviside step function, and $d>0$. The restoring force $f_{s}(\hat{x})$ is shown in Fig. 3. 


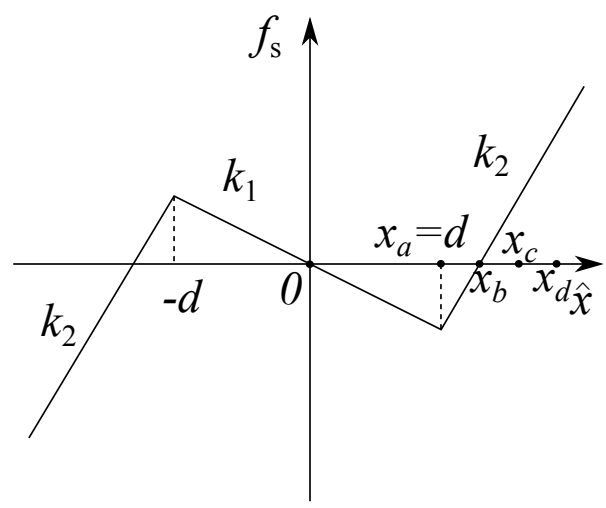

Fig. 3. Piecewise linear spring force with negative stiffness between $[-d, d]$.

The spring force has three linear segments. The middle segment, which is linear with negative stiffness, is centered at the origin, the two linear segments on the two sides have a same positive stiffness, and the whole force-displacement is centrally symmetric around the origin. It should be noted that the middle segment has negative stiffness rendering periodic solutions unstable within $[-d, d]$.

After the following transformation of space and time

$$
\begin{aligned}
& \hat{x}=x d, \\
& \hat{t}=t \sqrt{\frac{m}{k_{2}}},
\end{aligned}
$$

the original piecewise linear dynamic system Eq. (2) can be non-dimensionalized as

$$
\ddot{x}+\bar{\mu} \dot{x}+\bar{k} x+(1-\bar{k})[(x-1) H(x-1)+(x+1) H(-x-1)]=\bar{F} \cos \Omega t,
$$

where dot denotes differentiation with respect to the transformed time $t$, and

$$
\begin{aligned}
\bar{\mu} & =\frac{c}{\sqrt{k_{2} m}}>0 ; \\
\bar{k} & =\frac{k_{1}}{k_{2}}<0 \\
\bar{F} & =\frac{\hat{F}}{k_{2} d} \\
\Omega & =\hat{\Omega} \sqrt{\frac{m}{k_{2}}}
\end{aligned}
$$

Since Eq. (6) is obtained through linear transformation of time and space, i.e. Eqs. (4) and (5), the dynamic characteristics like stability and bifurcations of the original system Eq. (2) will not be changed essentially. Therefore, the dynamics of an arbitrary PWS system represented by Eq. (2) can totally be studied by investigating the corresponding non-dimensionalized Eq. (6).

In the next section, the dynamics of the non-dimensionalized oscillator Eq. (6) is analyzed based on numerical simulations, which is followed by two sections, where two cases are examined for Eq. (6): (1) $\bar{\mu}=0, \bar{F}=0$ and $(2) \bar{\mu}>0, \bar{F}>0$. Free vibration of the undamped system is first investigated with its exact solution derived. What follows is investigation of case (2). Frequency-response curves are obtained through numerical simulation for the forced and damped piecewise linear dynamic system, and compared to the first-order approximation obtained with the modified L-P method. 


\section{Numerical Investigations}

Existence of discontinuity in PWS dynamic systems causes inaccuracy during direct time integration, therefore accurate simulation of PWS dynamic systems requires specific numerical treatment [24]. Luo developed a theory of flow switchability to the boundaries in discontinuous dynamical systems [25]; the Gfunctions for piecewise smooth dynamic systems are introduced to analyze singularity in the systems, which provides an important method for the analysis of piecewise smooth dynamic systems. In addition, two other numerical techniques for simulating PWS systems are available [26, 27] and result in accurate simulations. One is to perform direct numerical simulation but with time and states resolved for any discontinuous points and obtain the periodic solutions of the PWS system by using event-driven simulation technique. The other is to obtain the time histories of the PWS system by using an LCP solver based on the time stepping method [10] in light of the fact that a PWS system is equivalent to an LCP. Both these algorithms take the motion switching into consideration in the discontinuous system and have been well studied, widely used, and can be used to simulate discontinuous dynamic systems accurately. It should be noted that all the numerical results in this section are obtained using an LCP solver based on the time stepping method.

\subsection{Phase-Plane Analysis}

For free vibration, a phase plane manifesting a set of initial conditions and the corresponding phase portraits is demonstrated in Fig. 4. The parameters in Eq. (6) held constant are the stiffness ratio, $\bar{k}=-2.0$, damping coefficient, $\bar{\mu}=0.15$, and excitation amplitude, $\bar{F}=0.0$.

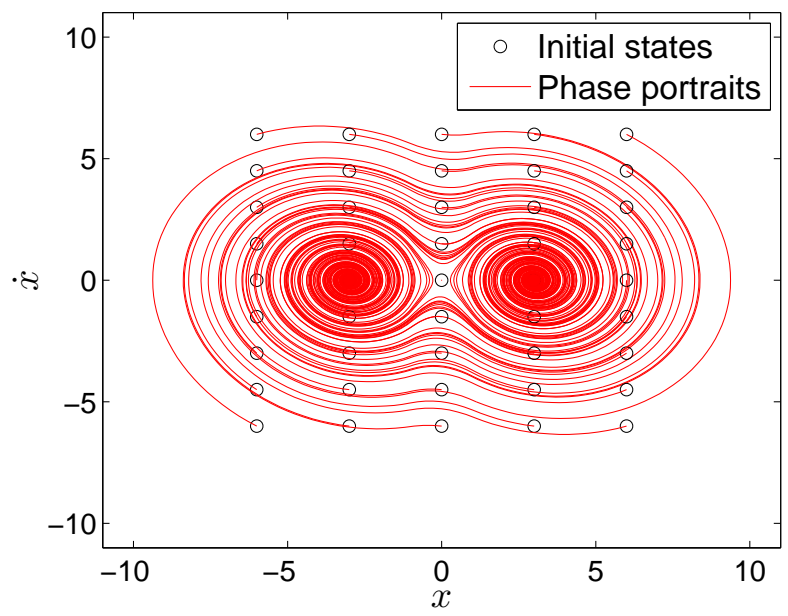

Fig. 4. Phase portraits of the free vibration system.

Fig. 4 suggests that when the oscillator is unforced and damped, its fixed points are the three zeros of the piecewise linear restoring force shown in Fig. 3, among which the origin is an unstable saddle point, while the two zeros on two sides are both stable foci.

For the forced and damped system, a phase plane with a set of initial conditions and the corresponding limit cycles is presented in Fig. 5. A couple of phase portraits are also furnished to show the general convergence process. The parameters held constant are the stiffness ratio, $\bar{k}=-2.0$, damping coefficient, $\bar{\mu}=0.05$, excitation amplitude, $\bar{F}=2.0$, and excitation frequency, $\Omega=0.6$.

It can be easily observed from Fig. 5 that under all the initial conditions, the oscillation converges to a same limit cycle. It is worth mentioning that some of the initial conditions given in Fig. 5 are near the initial conditions for two unstable periodic solutions. This suggests that only one stable periodic solution exists for the given set of system parameters. 


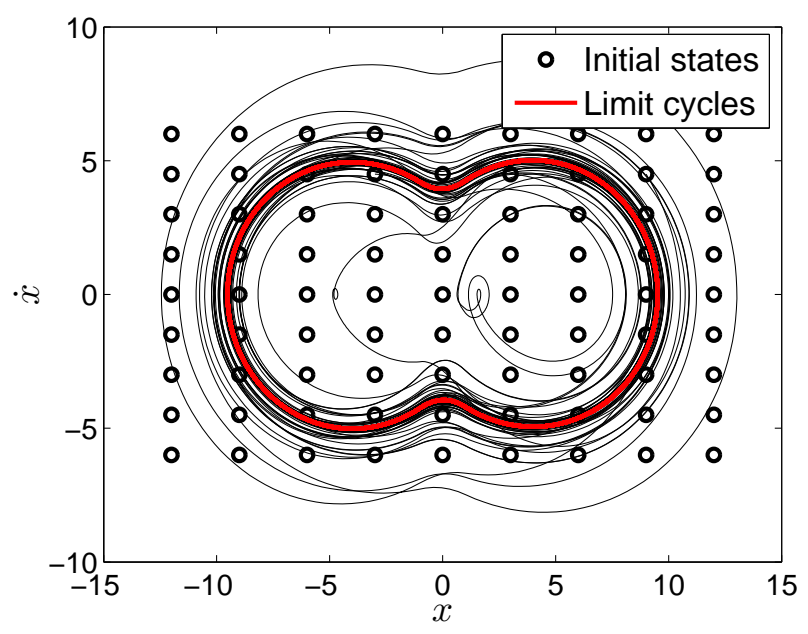

Fig. 5. Limit cycles with different initial conditions.

\subsection{Bifurcation Diagrams}

\subsection{1. $\bar{k}$ Variation}

Two bifurcation diagrams, which are shown in Fig. 6, have been constructed by changing the stiffness ratio, $\bar{k}$. The parameters held constant are excitation amplitude, $\bar{F}=1.0$, excitation frequency, $\Omega=0.4$, and damping coefficient differs for the two figures in Fig. 6.
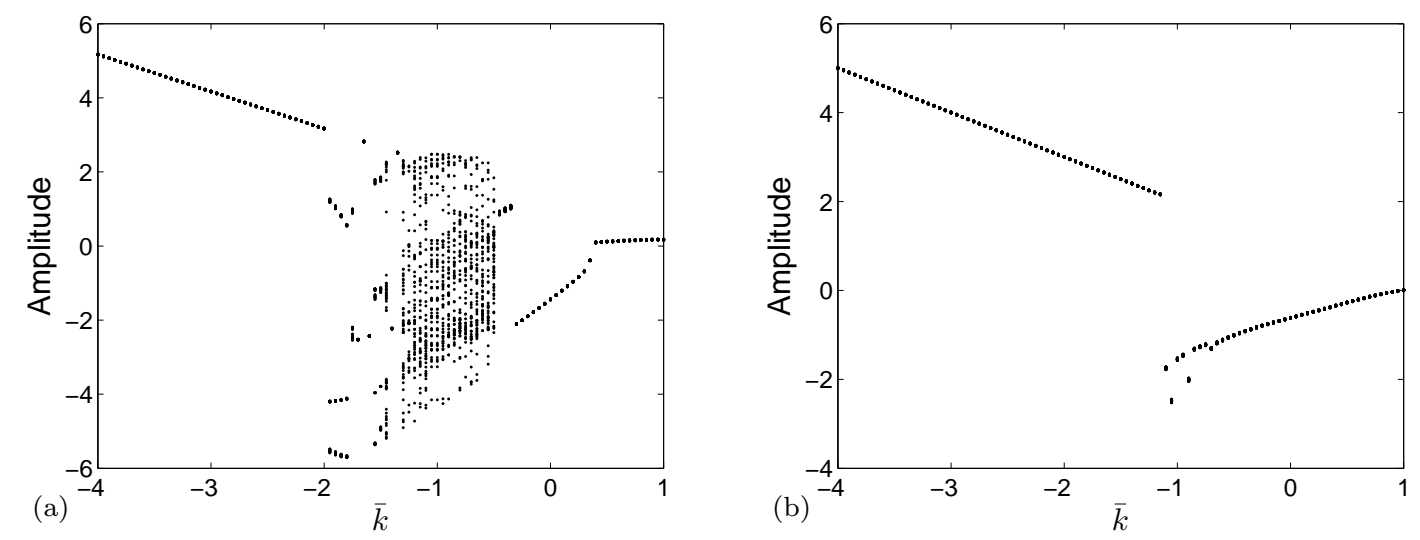

Fig. 6. Bifurcation diagram when $\bar{k}$ varies. (a) $\bar{\mu}=0.1$; (b) $\bar{\mu}=0.4$.

When $\bar{\mu}=0.1$, as seen from Fig. $6(\mathrm{a})$, regular periodic dynamics occur when $\bar{k}$ is close to zero. When $\bar{k}$ becomes smaller than -0.5 , the window of regular dynamics ends and potentially chaotic behavior arises; when $\bar{k}$ further decreases beyond -2.0 , an obvious period-one solution appears again. When $\bar{\mu}$ increases to 0.4 in Fig. 6(b), the region of potentially chaotic behavior is reduced by the adequate damping. This suggests that a damping adequate enough in dynamic system Eq. (6) can help control the chaos and keep the oscillation bounded. With the chaos controlled and the oscillation bounded, some good features of this piecewise linear system are presented for special cases. 


\subsection{2. $\bar{\mu}$ Variation}

By using the damping coefficient, $\bar{\mu}$, as the control parameter, a bifurcation diagram is constructed as shown in Fig. 7. The parameters held constant are the stiffness ratio, $\bar{k}=-4.0$, excitation amplitude, $\bar{F}=5.0$, and excitation frequency, $\Omega=1.2$.

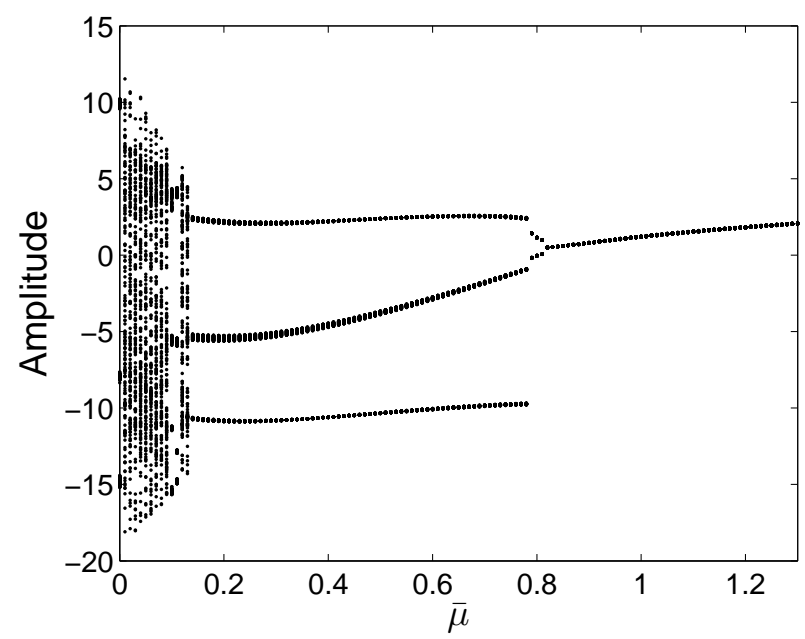

Fig. 7. Bifurcation diagram when $\bar{\mu}$ varies.

Figure 7 illustrates that when $\bar{\mu}$ is small, there exists interleaving of potential chaos and order. A little change in $\bar{\mu}$ can make a regular oscillation potentially chaotic. When $\bar{\mu}$ becomes large, the oscillation, however, exhibits regular periodic behavior only. Figure 7 further verifies the effect of damping on controlling chaotic behaviors in system Eq. (6).

\subsubsection{Excitation Amplitude Variation}

A bifurcation diagram with excitation amplitude, $\bar{F}$, as the control parameter, is shown in Fig. 8. In Fig. 8, the parameters held constant are the stiffness ratio $\bar{k}=-4.0$, damping coefficient $\bar{\mu}=0.2$, and excitation frequency $\Omega=1.2$.

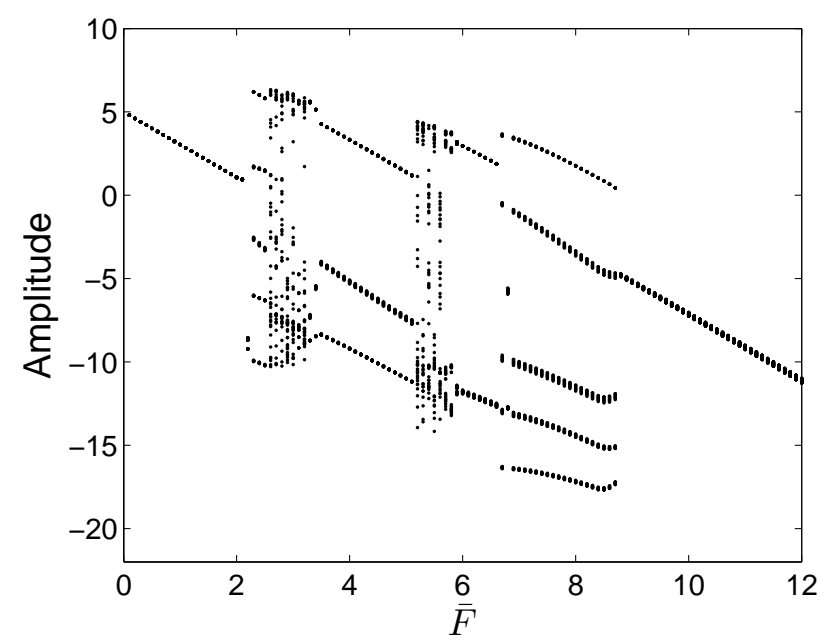

Fig. 8. Bifurcation diagram when $\bar{F}$ varies. 
Figure 8 shows that when $\bar{F}$ is very large, the oscillation exhibits regular periodic behavior. When $\bar{F}$ decreases, windows of potentially chaotic behavior alternate with those of regular periodic behavior. The vibration period can decrease or increase in multiples for small changes of $\bar{F}$ for this piecewise linear oscillator, which can be seen clearly around $\bar{F}=8.7$ in Fig. 8. And when $\bar{F}$ is close to zero, the steady-state response becomes regularly periodic again.

In summary, both very small excitation and very large excitation result in bounded periodic oscillation, and for middle-sized excitation, damping that is adequate enough can help reduce the chaotic behavior and keep the oscillation regular and bounded in special cases.

\subsection{Peak Amplitude and Peak Restoring Force}

With $\bar{F}=2.0$, the variation of peak amplitude versus $\bar{\mu}$ and $\bar{k}$ is depicted in Fig. 9(a). Figure 9(a) shows that when $\bar{\mu}$ increases from 0.02 to 0.34 , the resonance amplitude decreases drastically, while when $\bar{k}$ increases from -4.0 to 1.0 , the resonance amplitude decreases fairly slowly.
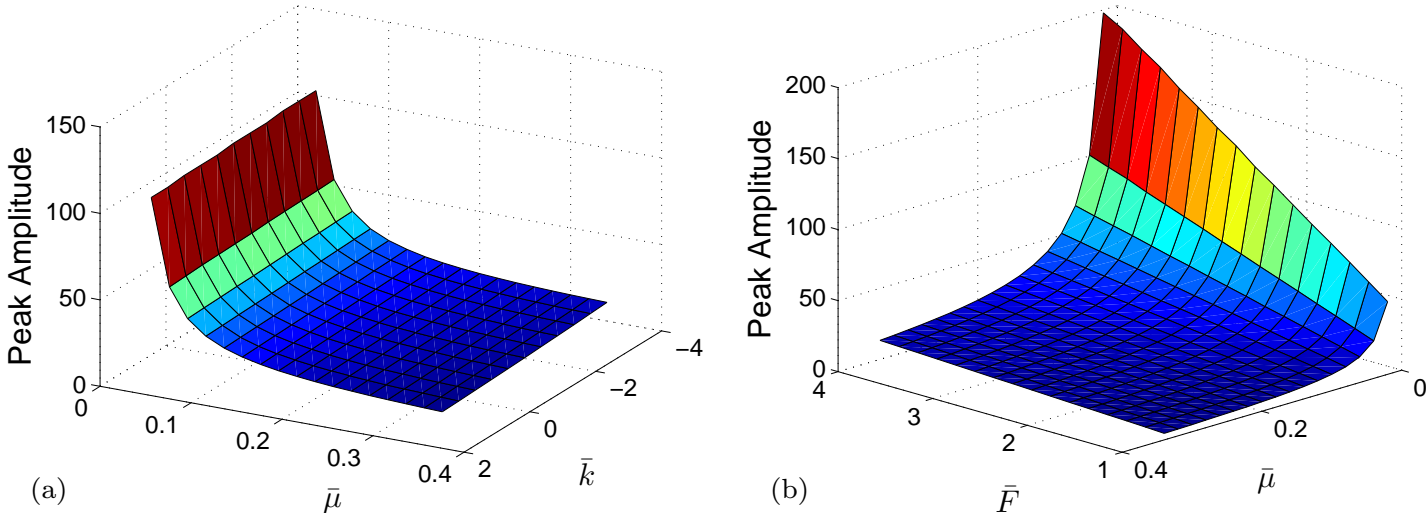

Fig. 9. Variation of resonance amplitude. (a) Versus $\bar{\mu}$ and $\bar{k}$; (b) versus $\bar{\mu}$ and $\bar{F}$.

With $\bar{k}=-1.0$, Fig. 9(b) presents the variation of peak amplitude versus $\bar{\mu}$ and $\bar{F}$. In Fig. 9(b), when $\bar{\mu}$ increases from 0.02 to 0.34 , the resonance amplitude first decreases drastically and then stabilizes, while when $\bar{k}$ increases from -4.0 to 1.0 , the resonance amplitude decreases relatively slowly.

Figure 10 presents four frequency response curves from Fig. 9(b). As the damping coefficient $\bar{\mu}$ increases from 0.05 to 0.4 , the peak amplitude drops quickly and the resonance frequency decreases.

Figure 11 depicts the relationship between the peak restoring force and the peak displacement amplitude when $\bar{k}$ changes. Demand or peak resonant response curves for different damping coefficients and capacity curves for different bilinear stiffness systems (with positive or negative initial stiffness) are depicted. Their intersection points represent the resonant displacement and restoring force for a particular. For example, point A in Fig. 11 represents a bilinear system with $\bar{k}$ of 6 and $\bar{\mu}$ of 0.10 , and point $\mathrm{C}$ represents a bilinear system with $\bar{k}$ of -1 and $\bar{\mu}$ of 0.20 . From Fig. 11, a negative $\bar{k}$ results in smaller restoring force than the purely linear case, i.e. $\bar{k}=1$, or a bilinear system with $\bar{k}=6$, at the price of larger peak displacement. Decreasing $\bar{k}$ from 6 to -1 for $\bar{\mu}$ of 0.10 can reduce the peak restoring force by about $40 \%$ (i.e. from point A to point $\mathrm{B}$ in Fig. 11). Corresponding system with decreasing $\bar{k}$ from 6 to -1 for $\bar{\mu}$ of 0.20 can reduce the peak restoring force by more than $50 \%$ (i.e. from point $\mathrm{D}$ to point $\mathrm{C}$ ). In addition, when $\bar{k}=6$ or -1 and increase in damping coefficient $\bar{\mu}$ from 0.10 to 0.20 , both the peak displacement and peak restoring force are reduced (i.e. from point A to point D or from point B to point C, respectively). Therefore, an appropriate combination of negative stiffness and large damping can reduce the peak restoring force with only a slightly larger peak displacement (i.e. from point A to point C). Essentially, the negative stiffness system in a dynamic system is very beneficial in reducing the amount of force transmitted. 

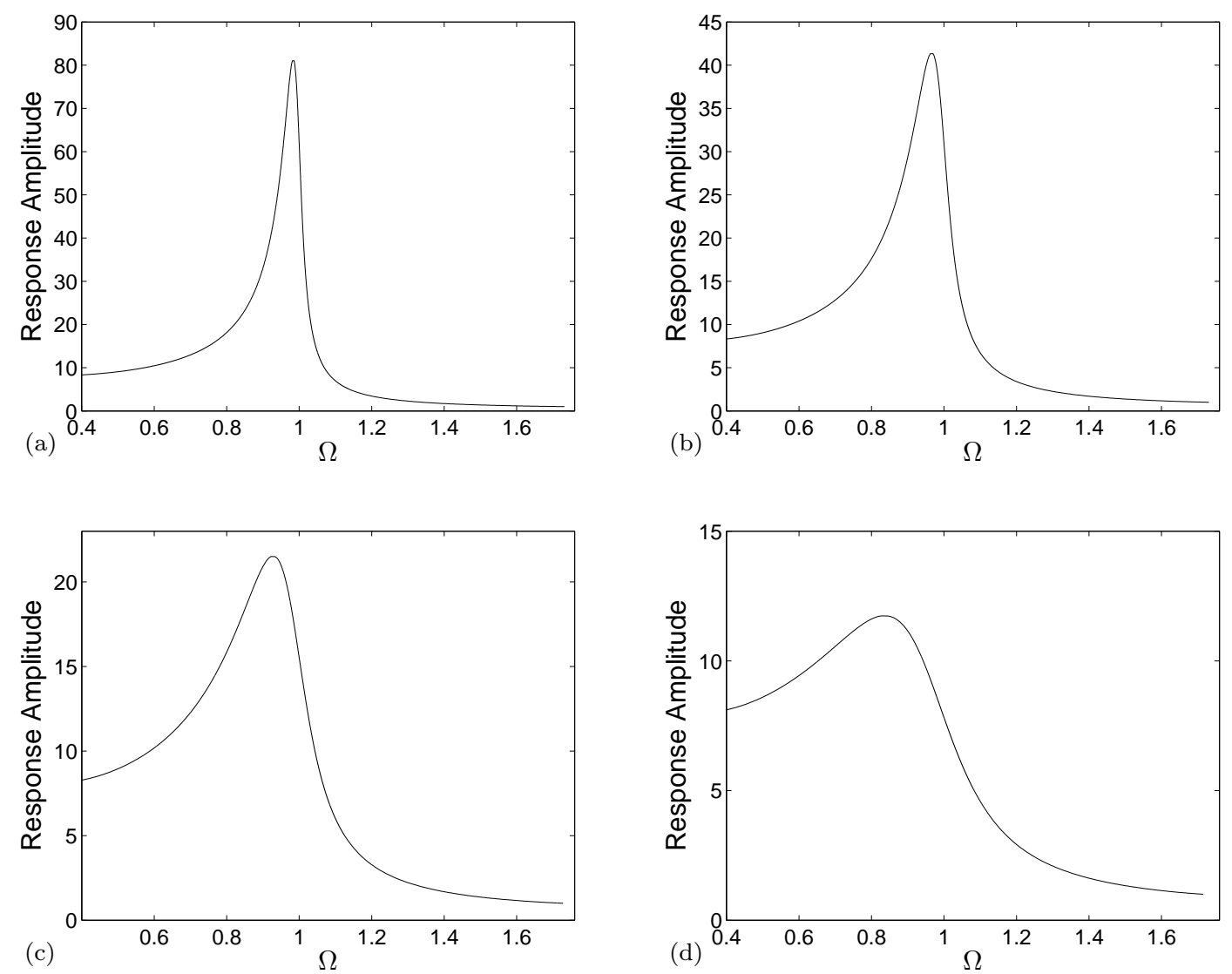

Fig. 10. Frequency response curves. (a) $\bar{\mu}=0.05, \bar{k}=-1.0, \bar{F}=4.0$; (b) $\bar{\mu}=0.1, \bar{k}=-1.0, \bar{F}=4.0$; (c) $\bar{\mu}=0.2, \bar{k}=-1.0, \bar{F}=4.0 ;$ (d) $\bar{\mu}=0.4, \bar{k}=-1.0, \bar{F}=4.0$.

In addition, Fig. 11 suggests that different combinations of stiffness and damping can be chosen according to the requirement of structural design. If the structure is designed for large force and small displacement amplitude, larger damping and larger negative stiffness or even positive stiffness can be chosen; point D in Fig. 11 represents a resultant performance under such a scenario. If the structure is designed for small force and large displacement amplitude, a combination of negative stiffness and large damping can be chosen; point $\mathrm{C}$ is an example of such a combination.

In summary, the existence of negative stiffness reduces the peak restoring force of the dynamic system Eq. (6) at the cost of increasing the peak displacement. Large damping can not only control chaos and ensure the oscillation bounded, but also reduces the peak restoring force and peak displacement simultaneously. Negative stiffness and large damping make a very good combination in dynamic systems because the force in the structure as well as the displacement amplitude can be reduced substantially.

In order to investigate the variation of the dynamics when $k_{1}$ and $k_{2}$ are varied (their difference remains the same), the following time transformation instead of Eq. (5) is performed

$$
\tilde{t}=t \sqrt{\frac{m}{\Delta k}},
$$

where $\Delta k=k_{2}-k_{1}$, while the space transformation is kept the same as Eq. (4), then the original piecewise 


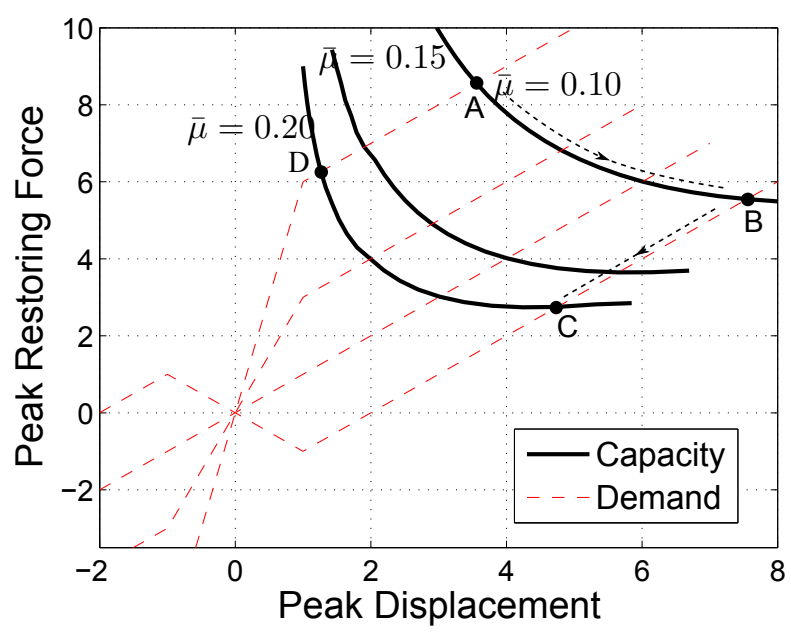

Fig. 11. The Trends of the Peak Restoring Force and the Peak Displacement Amplitude when $\bar{k}$ and $\bar{\mu}$ change.

linear dynamic system Eq. (2) can be non-dimensionalized as

$$
\frac{\mathrm{d}^{2} x}{\mathrm{~d} \tilde{t}^{2}}+\tilde{\mu} \frac{\mathrm{d} x}{\mathrm{~d} \tilde{t}}+\tilde{k} x+[(x-1) H(x-1)+(x+1) H(-x-1)]=\tilde{F} \cos \tilde{\Omega} \tilde{t} .
$$

where

$$
\begin{aligned}
\tilde{\mu} & =\frac{c}{\sqrt{\Delta k m}}>0 ; \\
\tilde{k} & =\frac{k_{1}}{\Delta k}<0 ; \\
\tilde{F} & =\frac{\hat{F}}{\Delta k d} ; \\
\tilde{\Omega} & =\hat{\Omega} \sqrt{\frac{m}{\Delta k}} .
\end{aligned}
$$

With $\tilde{F}=2.0$ in Eq. (12), the variation of peak amplitude versus $\tilde{\mu}$ and $\tilde{k}$ is depicted in Fig. 12. Figure 12 shows that when $\tilde{\mu}$ increases from 0.02 , the resonance amplitude decreases drastically at first, and then becomes much more stable after 0.2 . When $\tilde{k}$ increases from -0.8 to 0 , the resonance amplitude decreases fast only for small damping. For large damping, the resonance amplitude decreases very slowly. Variation of $\tilde{k}$ can be interpreted as changing $k_{1}$ with $\Delta k$ unchanged in Eq. (14); then the variation of response amplitude as function of $\tilde{k}$ is shown in Fig. 12. Change in $k_{1}$ results in an equal change in $k_{2}\left(k_{2}=k_{1}+\Delta k\right)$.

\subsection{Time Histories and Dynamic Characteristics}

Figure 13 shows time history and phase portrait, which are obtained using an LCP solver based on time stepping method for $\bar{\mu}=0.05, \bar{k}=-3.0, \bar{F}=2.0$, and $\Omega=0.9$.

There are three equilibrium points for the dynamic system represented by Eq. (6). One is the origin, which is unstable, and the other two are the two intersection points on the displacement axis on the two sides, which are stable equilibrium points. Figure 14(a) gives an example that the oscillation converges to a limit cycle around one stable equilibrium point other than the origin for certain initial condition, which coincides with the phenomenon observed in experiment [28]. Figure 14(b) presents the fluctuation of mechanical energy as displacement and velocity evolve. 


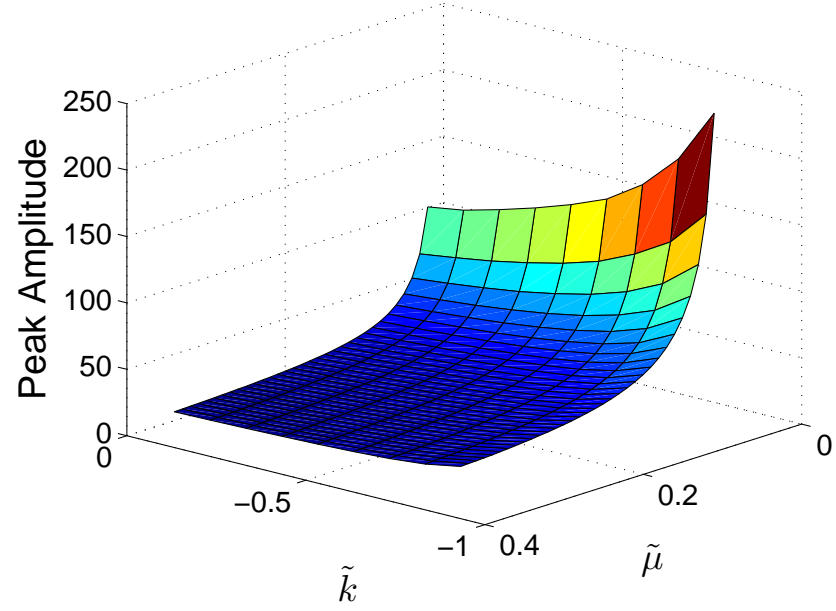

Fig. 12. Variation of Resonance Amplitude versus $\tilde{\mu}$ and $\tilde{k}$.
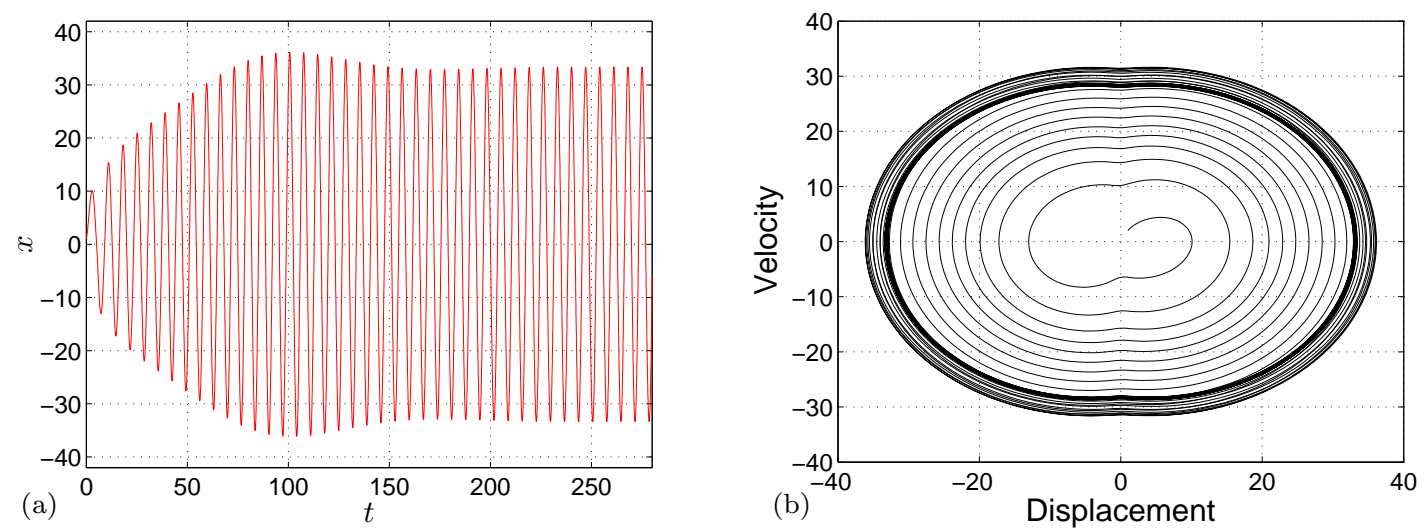

Fig. 13. Simulation Results for $\bar{\mu}=0.05, \bar{k}=-3.0, \bar{F}=2.0$, and $\Omega=0.9$. (a) Time history; (b) phase portrait.

Figure 15 indicates that the oscillations initially increase drastically because of the negative stiffness segment, after which a piecewise linear system with segments of positive stiffness on two sides confines the motion and oscillations are bounded eventually reaching a limit cycle. Note the transient behavior of the oscillations before the limit cycle. Since the frequency of excitation is in the post resonance period, the limit cycle response is smaller than the transient response. It should be noted van der Pol oscillator, which is another nonlinear system with double-well potential, also has this similar dynamic characteristic. All the time histories in Figs. 13, 14, and 15, are obtained by using an LCP solver based on time stepping method.

\section{FREE VIBRATION OF THE UNDAMPED SYSTEM: $\bar{\mu}=0, \bar{F}=0$}

Let $\bar{\mu}=0, \bar{F}=0$, then the non-dimensionalized dynamic system (6) reduces to

$$
\ddot{x}+\bar{k} x+(1-\bar{k})[(x-1) H(x-1)+(x+1) H(-x-1)]=0 .
$$

The exact solution as well as vibration period of Eq. (17) can be readily calculated analytically. Assuming 

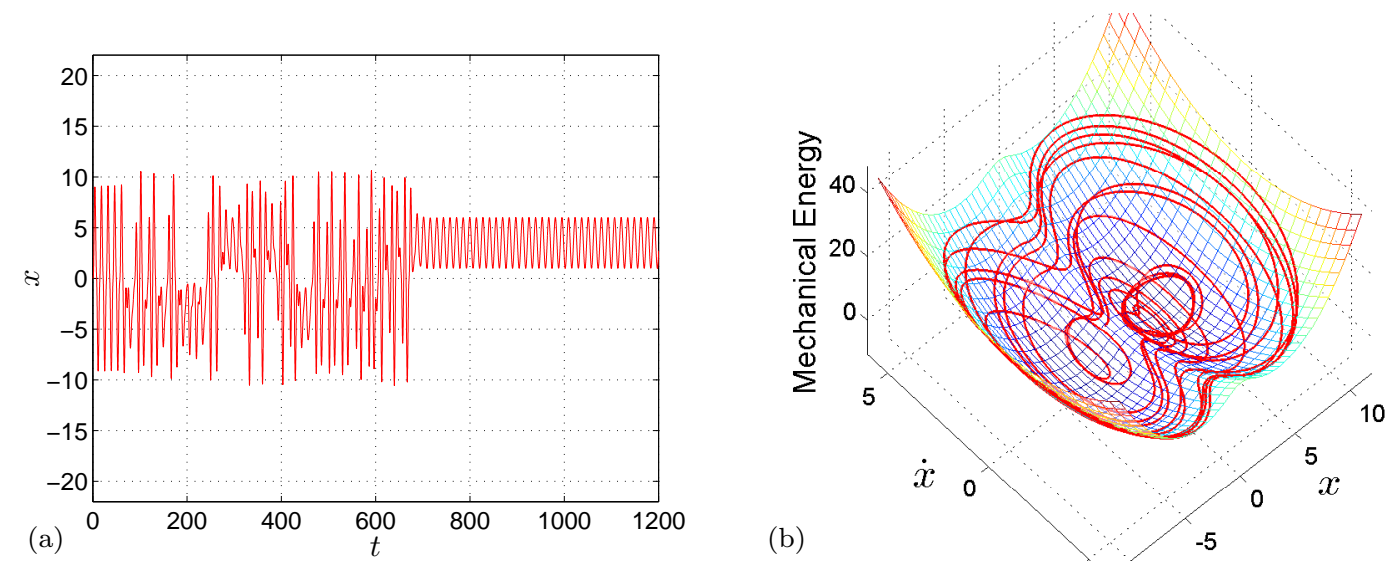

Fig. 14. Simulation Results for $\bar{\mu}=0.18, \bar{k}=-2.5, \bar{F}=2.0$, and $\Omega=0.45$. (a) Time history; (b) phase portrait on the mechanical energy surface.
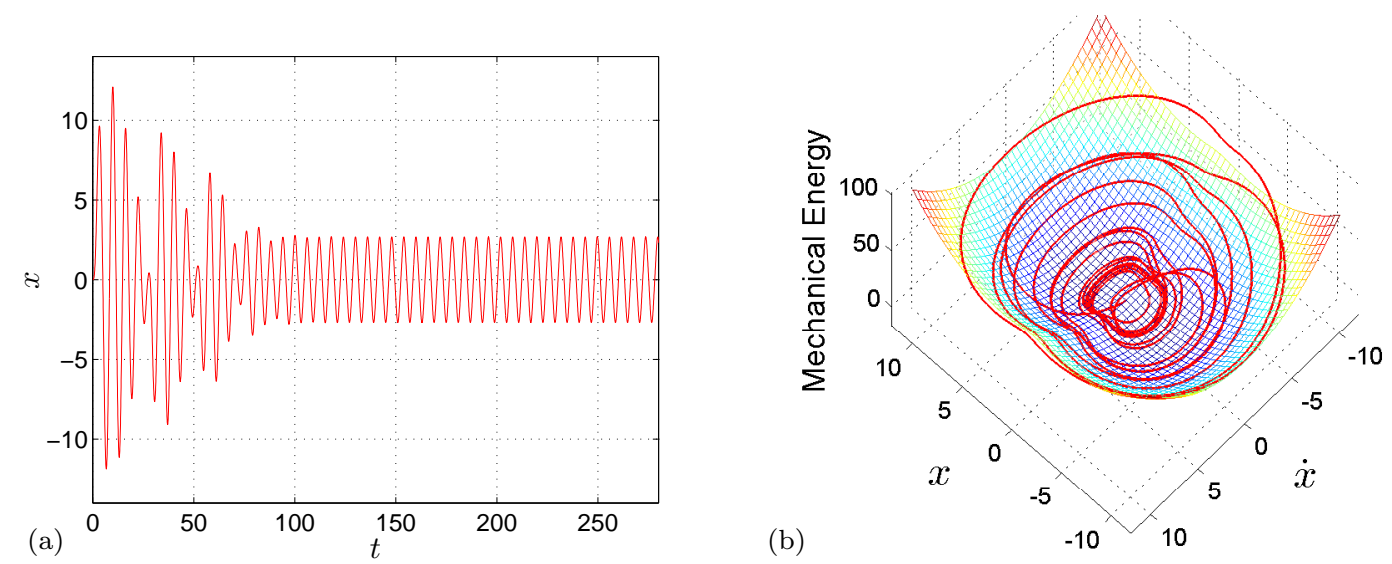

Fig. 15. Time History of a Piecewise Linear System with $\bar{\mu}=0.2, \bar{k}=-2.0, \bar{F}=4.0$, and $\Omega=1.05$. (a) Time history; (b) phase portrait on the mechanical energy surface.

that the initial displacement is $A_{e}$ and the initial velocity is 0 , we only consider the cases for $A_{e} \geq 0$ since the cases for $A_{e} \leq 0$ can be easily obtained according to the symmetry of the dynamic system.

The potential energy for Eq. (17) is

$$
\begin{aligned}
G(x) & =\int_{0}^{x}\{\bar{k} x+(1-\bar{k})[(x-1) H(x-1)+(x+1) H(-x-1)]\} \mathrm{d} x \\
& = \begin{cases}\frac{1}{2} \bar{k} x^{2}, & -1 \leq x \leq 1, \\
\frac{1}{2} \bar{k} x^{2}+\frac{1}{2}(1-\bar{k})(|x|-1)^{2}, & |x|>1,\end{cases}
\end{aligned}
$$

then the energy equation shall be

$$
E(t)=\frac{v^{2}}{2}+G(x)=E_{0},
$$

where $v$ is the velocity of the oscillator, and $E_{0}$ is a constant representing the total mechanical energy.

One critical point lies beyond the zero point $x_{b}=1-\bar{k}$ (see Fig. 3), where $G\left(x_{d}\right)=G(0)=0$. It is quite easy to obtain $x_{d}=1-\bar{k}+\sqrt{-\bar{k}(1-\bar{k})}$. Another three critical points are the origin, $x_{a}=1$ and 
$x_{c}=1-2 \bar{k}$. When $A_{e} \in(0,1)$ or $A_{e} \in\left(x_{c}, x_{d}\right)$, the oscillator runs over two segments of the piecewise linear restoring force; when $A_{e} \in\left[1, x_{c}\right]$, the oscillation becomes purely harmonic about $x_{b}=1-\bar{k}$ within the rightmost segment of the piecewise linear restoring force, which will not be considered hereafter since the system becomes linear under this case; when $A_{e} \in\left(x_{d}, \infty\right)$, a periodic oscillation running over all the three segments occurs.

First, we consider the case for $A_{e} \in(0,1)$ or $A_{e} \in\left(x_{c}, x_{d}\right)$. We only need to consider the case for $A_{e} \in(0,1)$, since an oscillation starting from a point $\left(A_{e}, 0\right), A_{e} \in(0,1)$ in the phase plane will pass through $\left(A_{e}^{\prime}, 0\right), A_{e}^{\prime} \in\left(x_{c}, x_{d}\right)$, and vice versa. Starting from $\left(A_{e}, 0\right), A_{e} \in(0,1)$, before $x$ reaches $A_{e}^{\prime}$,

$$
E_{0}=\frac{v^{2}}{2}+G(x)=G\left(A_{e}\right)=\frac{1}{2} \bar{k} A_{e}^{2}
$$

Therefore,

$$
v(x)=\sqrt{2\left[G\left(A_{e}\right)-G(x)\right]}= \begin{cases}\sqrt{\bar{k}\left(A_{e}^{2}-x^{2}\right)}, & 0<x<1, \\ \sqrt{\bar{k}\left(A_{e}^{2}+1-2 x\right)-(x-1)^{2}}, & 1<x<A_{e}^{\prime}\end{cases}
$$

where $G(x)$ is given by Eq. (18).

It follows from Eq. (21) that the time required for the representative point to travel in the upper half phase plane from $\left(A_{e}, 0\right)$ to an arbitrary point of abscissa $x$ is

$$
\begin{aligned}
t(x) & =\int_{A_{e}}^{x} \frac{\mathrm{d} x}{v(x)} \\
& = \begin{cases}\int_{A_{e}}^{x} \frac{\mathrm{d} x}{\sqrt{\bar{k}\left(A_{e}^{2}-x^{2}\right)}}, & 0<x \leq 1, \\
\int_{A_{e}}^{1} \frac{\mathrm{d} x}{\sqrt{\bar{k}\left(A_{e}^{2}-x^{2}\right)}}+\int_{1}^{x} \frac{\mathrm{d} x}{\sqrt{\bar{k}\left(A_{e}^{2}+1-2 x\right)-(x-1)^{2}}}, & 1<x<A_{e}^{\prime}\end{cases} \\
& = \begin{cases}\frac{1}{\sqrt{-\bar{k}}} \ln \frac{x+\sqrt{x^{2}-A_{e}^{2}}}{A_{e}}, & 0<x \leq 1, \\
\frac{1}{\sqrt{-\bar{k}}} \ln \frac{1+\sqrt{1-A_{e}^{2}}}{A_{e}}+\arccos \frac{\bar{k}}{\sqrt{\bar{k}\left[A_{e}^{2}-(1-\bar{k})\right]}} & 1<x<A_{e}^{\prime} . \\
-\arccos \frac{x-(1-\bar{k})}{\sqrt{\bar{k}\left[A_{e}^{2}-(1-\bar{k})\right]},}\end{cases}
\end{aligned}
$$

Then based on Eq. (22), it is straightforward to obtain the displacement of the oscillator after solving linear differential equations.

$$
x(t)= \begin{cases}\frac{A_{e}}{2}\left(e^{\sqrt{-\bar{k}} t}+e^{-\sqrt{-\bar{k}} t}\right), & 0<t \leq t_{1}, \\ (1-\bar{k})-B \cos \left[\left(t-t_{1}\right)+\arccos \left(-\frac{\bar{k}}{B}\right)\right], & t_{1}<t<t_{2},\end{cases}
$$

where

$$
\begin{aligned}
t_{1} & =\frac{1}{\sqrt{-\bar{k}}} \ln \frac{1+\sqrt{1-A_{e}^{2}}}{A_{e}} \\
B & =\sqrt{\bar{k}\left[A_{e}^{2}-(1-\bar{k})\right]} \\
t_{2} & =\frac{1}{\sqrt{-\bar{k}}} \ln \frac{1+\sqrt{1-A_{e}^{2}}}{A_{e}}+2 \arccos \frac{\bar{k}}{B} .
\end{aligned}
$$

According to the symmetry of the oscillation, the period is given by

$$
T\left(A_{e}\right)=2 t_{2}=\frac{2}{\sqrt{-\bar{k}}} \ln \frac{1+\sqrt{1-A_{e}^{2}}}{A_{e}}+4 \arccos \frac{\bar{k}}{B} .
$$



0 ,

Secondly, we consider the case for $A_{e} \in\left(x_{d}, \infty\right)$. Starting from $\left(A_{e}, 0\right), A_{e} \in\left(x_{d}, \infty\right)$, before $x$ reaches

$$
E_{0}=\frac{v^{2}}{2}+G(x)=G\left(A_{e}\right)=\frac{1}{2} A_{e}^{2}+\frac{1}{2}(1-\bar{k})\left(1-2 A_{e}\right) .
$$

Hence

$$
v(x)=-\sqrt{2\left(G\left(A_{e}\right)-G(x)\right)}= \begin{cases}-\sqrt{\left[A_{e}-(1-\bar{k})\right]^{2}+\bar{k}(1-\bar{k})-\bar{k} x^{2}}, & 0<x \leq 1, \\ -\sqrt{\left[A_{e}-(1-\bar{k})\right]^{2}-[x-(1-\bar{k})]^{2}}, & x>1,\end{cases}
$$

where $G(x)$ is given by Eq. (18).

It follows from Eq. (21) the time required for the representative point to travel in the lower half phase plane from $\left(A_{e}, 0\right)$ to an arbitrary point of abscissa $x$ is

$$
\begin{aligned}
t(x) & =\int_{A_{e}}^{x} \frac{\mathrm{d} x}{v(x)} \\
& = \begin{cases}\frac{1}{\sqrt{-\bar{k}}} \ln \frac{\sqrt{-\bar{k}}+\sqrt{\hat{A}_{e}^{2}-\bar{k}^{2}}}{\sqrt{-\bar{k}} x+\sqrt{-\bar{k} x^{2}+\hat{A}_{e}^{2}+\bar{k}(1-\bar{k})}}+\arccos \frac{\bar{k}}{\hat{A}_{e}}, & 0 \leq x \leq 1, \\
\arccos \frac{x-(1-\bar{k})}{\hat{A}_{e}}, & 1<x \leq A_{e},\end{cases}
\end{aligned}
$$

where

$$
\hat{A}_{e}=A_{e}-(1-\bar{k}) .
$$

Based on Eq. (30), the displacement of the oscillator can be easily obtained through solving linear differential equations.

$$
x(t)= \begin{cases}c_{1} e^{-\sqrt{-\bar{k}} t}+c_{2} e^{\sqrt{-\bar{k}} t}, & t_{3}<t \leq t_{4}, \\ \hat{A}_{e} \cos t+(1-\bar{k}), & 0<t \leq t_{3},\end{cases}
$$

where

$$
\begin{aligned}
t_{3} & =t(1)=\arccos \frac{\bar{k}}{\hat{A}_{e}}, \\
t_{4} & =t(0)=t_{3}+\frac{1}{\sqrt{-\bar{k}}} \ln \frac{\sqrt{-\bar{k}}+\sqrt{\hat{A}_{e}^{2}-\bar{k}^{2}}}{\sqrt{\hat{A}_{e}^{2}+\bar{k}(1-\bar{k})}}, \\
c_{1} & =\frac{\sqrt{-\bar{k}}+\hat{A}_{e} \sin t_{3}}{2 \sqrt{-\bar{k}} e^{-\sqrt{-\bar{k}} t_{3}}} \\
c_{2} & =\frac{\sqrt{-\bar{k}}-\hat{A}_{e} \sin t_{3}}{2 \sqrt{-\bar{k}} e^{\sqrt{-\bar{k}} t_{3}}} .
\end{aligned}
$$

According to the symmetry of the oscillation, the period is governed by

$$
T\left(A_{e}\right)=4 t_{4}=4 \arccos \frac{\bar{k}}{\hat{A}_{e}}+\frac{4}{\sqrt{-\bar{k}}} \ln \frac{\sqrt{-\bar{k}}+\sqrt{\hat{A}_{e}^{2}-\bar{k}^{2}}}{\sqrt{\hat{A}_{e}^{2}+\bar{k}(1-\bar{k})}} .
$$

By setting $\bar{k}=-2.0$, the vibration period given by Eq. (37) is presented in Fig. 16. Figure 16 illustrates asymptotic behavior of the period. 


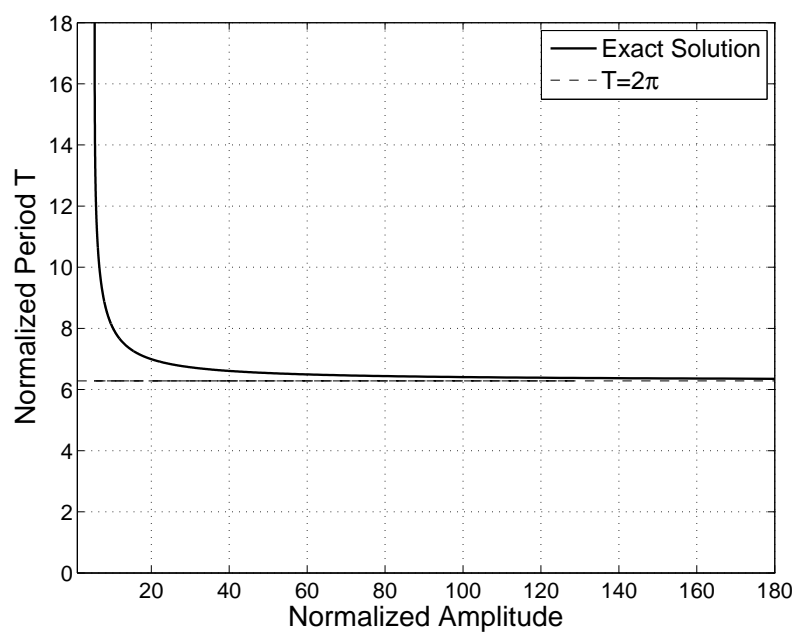

Fig. 16. Period-amplitude curves of the exact solution.

\section{FORCED AND DAMPED SYSTEM: $\bar{\mu}>0, \overline{\boldsymbol{F}}>0$}

\subsection{Frequency-Response Curves and Limit Cycles based on Numerical Simulation}

Let $\bar{\mu}=0.05$ and $\bar{F}=2.0$, varying $\bar{k}$, we numerically obtain the frequency-response curves in Fig. 17 (all the lines other than the circles) by using an algorithm based on a continuation method [29] and an event-driven simulation technique [9].

When $\bar{k}$ is small in absolute value as in Fig. 17(a), the frequency-response curve does not exhibit obvious hardening or softening trend. When the absolute value of $\bar{k}$ increases, a hardening trend develops, while the peak amplitude of the frequency-response curve increases very slowly as seen in Fig. 17(a) to Fig. 17(d). When $\bar{k}=-2.0$, the upper branch starts to form a loop as seen in Fig. 17(c). As $|\bar{k}|$ further increases, the loop becomes totally detached from the lower unstable branch, which is depicted as the lower dashed line in Fig. 17(d). As long as the response amplitude is smaller than 1, only unstable periodic solution exists because only negative stiffness is functioning. As $\bar{k}$ changes throughout Fig. 17, the peak amplitude of the frequency response curve stays at almost the same magnitude and occurs always near the nondimensionalized frequency one, which means that for the given magnitude of excitation, $\bar{k}$ has little effect on the resonance amplitude or frequency.

In Fig. 17, note that the dashed lines with amplitude lower than 1, which correspond to unstable periodic solutions of the oscillation within the middle segment shown in Fig. 3, are calculated exactly; while, only the dashed and solid lines above 1 are obtained using event-driven simulation technique. In Figs. 17(a), (b) and (c), the lines obtained through exact calculation and simulation connect with each other where the amplitude is exactly 1 , which verifies the correctness of the simulation results. In Fig. 17(c), where the dashed line below 1 reaches 1 at 0 excitation frequency, the closed loop starts to arise. It should be noted that Fig. 17 (c) is in good agreement with Fig. 5 in that the response amplitudes at $\Omega=0.6$ match with each other and only one stable periodic solution exists for the given set of system parameters. Moreover, the peak amplitude at $\Omega=0.9$ in Fig. 17(d) is in good agreement with Fig. 13(a). Therefore, the numerical results obtained through the event-driven algorithm and those through the LCP solver verify the validity of each other.

\subsection{Approximation with a Modified Lindstedt-Poincaré Method}

In what follows, as two discontinuous points arise and damping and excitation terms exist at the same time, a modified Lindstedt-Poincaré method [29] is adopted to solve Eq. (6). 

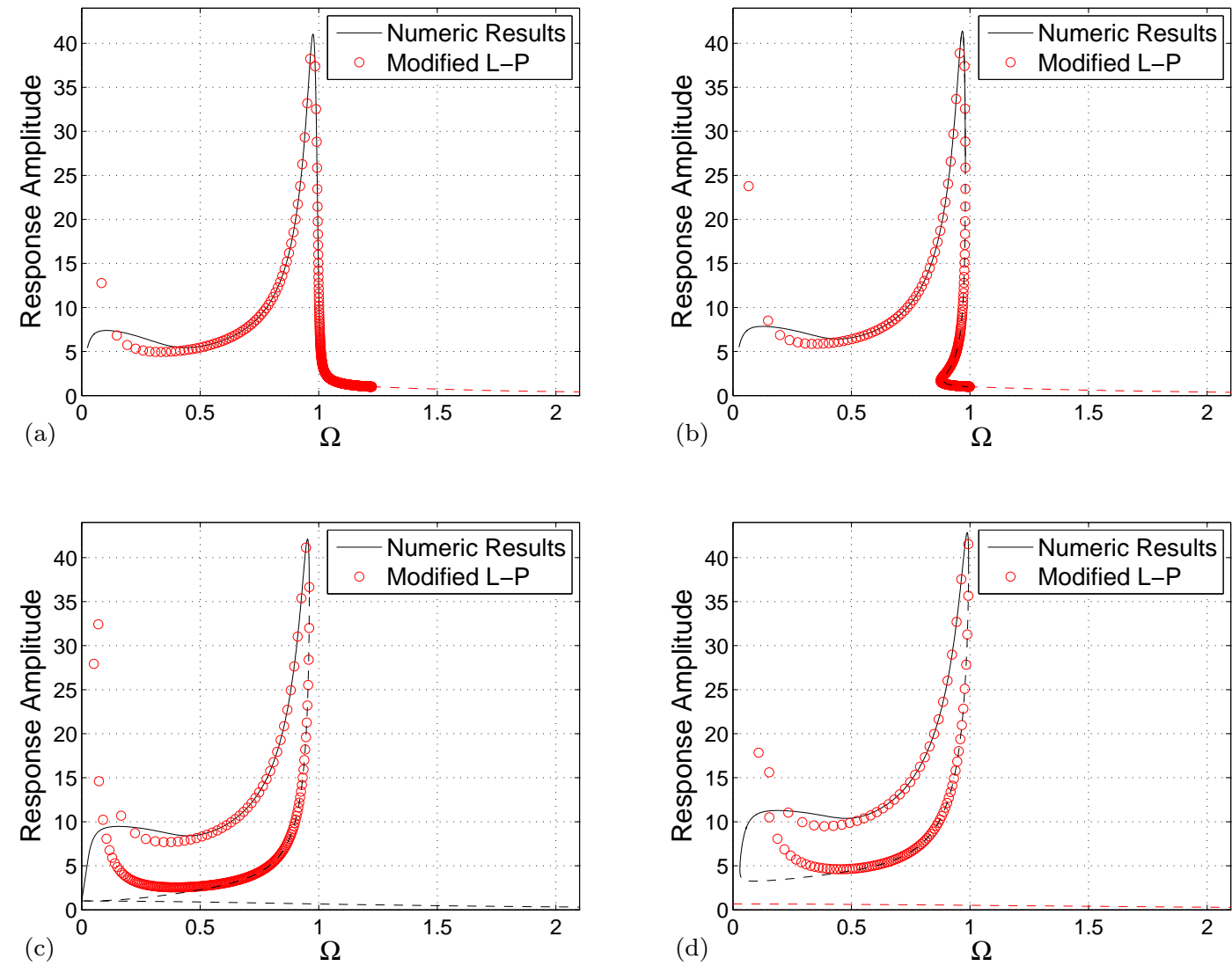

Fig. 17. Forced and damped frequency-response curves when $\bar{k}$ increases(solid line: stable solution; dashed line: unstable solution). (a) $\bar{k}=-0.5$; (b) $\bar{k}=-1.0$; (c) $\bar{k}=-2.0$; (d) $\bar{k}=-3.0$.

We first substitute $\bar{\mu}=\varepsilon \mu, \bar{k}=1-\varepsilon k$ and $\bar{F}=\varepsilon F$ into Eq. (6) so that the variable $\varepsilon>0$ serves as a measure of the parameters' magnitude, and then Eq. (6) can be rewritten as

$$
\ddot{x}+\varepsilon \mu \dot{x}+(1-\varepsilon k) x+\varepsilon k[(x-1) H(x-1)+(x+1) H(-x-1)]=\varepsilon F \cos \Omega t .
$$

We then conduct a linear time transformation $\tau=\Omega t$ in Eq. (38) and it becomes

$$
\Omega^{2} x^{\prime \prime}+\varepsilon \mu \Omega x^{\prime}+(1-\varepsilon k) x+\varepsilon k[(x-1) H(x-1)+(x+1) H(-x-1)]=\varepsilon F \cos \tau,
$$

where the prime symbol denotes differentiation with respect to $\tau$.

It has been found that expanding $\Omega^{2}$ other than $\Omega$ is important for adapting Lindstedt-Poincaré method to large parameters in ODEs, therefore $\Omega^{2}$ is expanded as follows.

$$
\Omega^{2}=1+\sum_{n=1}^{\infty} \varepsilon^{n} \omega_{n}
$$

where $\omega_{n}, n=1,2, \cdots$, are to be determined.

Define a new parameter

$$
\alpha=\frac{\varepsilon \omega_{1}}{1+\varepsilon \omega_{1}},
$$


such that

$$
\varepsilon=\frac{\alpha}{\omega_{1}(1-\alpha)},
$$

and hence

$$
\begin{aligned}
\Omega^{2} & =\left(1+\varepsilon \omega_{1}\right)\left[1+\frac{1}{1+\varepsilon \omega_{1}}\left(\varepsilon^{2} \omega_{2}+\varepsilon^{3} \omega_{3}+\cdots\right)\right] \\
& =\frac{1}{1-\alpha}\left(1+\delta_{2} \alpha^{2}+\delta_{3} \alpha^{3}+\cdots\right),
\end{aligned}
$$

where $\delta_{n}, n=2,3, \cdots$, are unknowns to be determined in the later secular term elimination steps.

Substituting Eqs. (40), (42), and (43) into Eq. (39), we have

$$
\begin{aligned}
\left(1+\delta_{2} \alpha^{2}+\delta_{3} \alpha^{3}\right. & +\cdots) x^{\prime \prime}+\frac{\mu \Omega \alpha}{\omega_{1}} x^{\prime}+\left[1-\left(1+\frac{k}{\omega_{1}}\right) \alpha\right] x \\
& +\frac{\alpha}{\omega_{1}} k[(x-1) H(x-1)+(x+1) H(-x-1)]=\frac{F \alpha}{\omega_{1}} \cos \tau .
\end{aligned}
$$

Assuming the solution of Eq. (44) can be expanded as

$$
x=x_{0}+\alpha x_{1}+\alpha^{2} x_{2}+\cdots
$$

Substituting Eq. (45) into Eq. (44), then collecting the terms with the identical powers of $\alpha$, one has

$$
\begin{aligned}
\alpha^{0} \quad: \quad & x_{0}^{\prime \prime}+x_{0}=0, \\
\alpha^{1}: & x_{1}^{\prime \prime}+x_{1}=\frac{F}{\omega_{1}} \cos \tau-\frac{\mu \Omega}{\omega_{1}} x_{0}^{\prime}+\left(1+\frac{k}{\omega_{1}}\right) x_{0} \\
\quad & \quad-\frac{k}{\omega_{1}}\left[\left(x_{0}-1\right) H\left(x_{0}-1\right)+\left(x_{0}+1\right) H\left(-x_{0}-1\right)\right], \\
\alpha^{2}: & \quad x_{2}^{\prime \prime}+x_{2}=-\delta_{2} x_{0}^{\prime \prime}-\frac{\mu \Omega}{\omega_{1}} x_{1}^{\prime}+\left(1+\frac{k}{\omega_{1}}\right) x_{1} \\
& \quad-\frac{k x_{1}}{\omega_{1}}\left[H\left(x_{0}-1\right)+H\left(-x_{0}-1\right)+\left(x_{0}-1\right) \delta\left(x_{0}-1\right)-\left(x_{0}+1\right) \delta\left(-x_{0}-1\right)\right],
\end{aligned}
$$

where $\delta(\cdot)$ is the Dirac delta function.

The solution to Eq. (46) is

$$
x_{0}=A_{0} \cos \left(\tau+\phi_{0}\right) \text {, }
$$

where $A_{0} \geq 1$.

Substitution of Eq. (49) into Eq. (47) yields

$$
\begin{aligned}
x_{1}^{\prime \prime}+x_{1}= & \frac{F}{\omega_{1}} \cos \tau+\frac{\mu \Omega}{\omega_{1}} A_{0} \sin \left(\tau+\phi_{0}\right)-\frac{k}{\omega_{1}}\left\{\left[A_{0} \cos \left(\tau+\phi_{0}\right)-1\right] H\left(A_{0} \cos \left(\tau+\phi_{0}\right)-1\right)\right. \\
& \left.+\left[A_{0} \cos \left(\tau+\phi_{0}\right)+1\right] H\left(-A_{0} \cos \left(\tau+\phi_{0}\right)-1\right)\right\}+\left(1+\frac{k}{\omega_{1}}\right) A_{0} \cos \left(\tau+\phi_{0}\right) .
\end{aligned}
$$

Because $H\left(A_{0} \cos \left(\tau+\phi_{0}\right)-1\right)$ and $H\left(-A_{0} \cos \left(\tau+\phi_{0}\right)-1\right)$ are periodic function with circular frequency of 1 , they can be expanded as the following Fourier series.

$$
\begin{aligned}
H\left(A_{0} \cos \left(\tau+\phi_{0}\right)-1\right) & =\frac{a_{0}}{2}+\sum_{n=1}^{\infty} a_{n} \cos n\left(\tau+\phi_{0}\right), \\
H\left(-A_{0} \cos \left(\tau+\phi_{0}\right)-1\right) & =\frac{a_{0}}{2}+\sum_{n=1}^{\infty}(-1)^{n} a_{n} \cos n\left(\tau+\phi_{0}\right),
\end{aligned}
$$


where $a_{n}=\frac{2 \sin n \theta_{0}}{n \pi},(n=0,1,2, \ldots)$ and $\theta_{0}=\arccos \frac{1}{A_{0}} \in\left[0, \frac{\pi}{2}\right)$.

Substitution of Eq. (51) and Eq. (52) into Eq. (50) gives

$$
\begin{aligned}
x_{1}^{\prime \prime}+x_{1}= & \frac{F}{\omega_{1}} \cos \tau+\frac{\mu \Omega}{\omega_{1}} A_{0} \sin \left(\tau+\phi_{0}\right)-\frac{k}{\omega_{1}}\left\{\left[A_{0} \cos \left(\tau+\phi_{0}\right)-1\right]\left[\frac{a_{0}}{2}+\sum_{n=1}^{\infty} a_{n} \cos n\left(\tau+\phi_{0}\right)\right]\right. \\
& \left.+\left[A_{0} \cos \left(\tau+\phi_{0}\right)+1\right]\left[\frac{a_{0}}{2}+\sum_{n=1}^{\infty}(-1)^{n} a_{n} \cos n\left(\tau+\phi_{0}\right)\right]\right\}+\left(1+\frac{k}{\omega_{1}}\right) A_{0} \cos \left(\tau+\phi_{0}\right) \\
= & \frac{F}{\omega_{1}} \cos \tau-\frac{k}{\omega_{1}}\left\{a_{0} A_{0} \cos \left(\tau+\phi_{0}\right)+\sum_{n=1}^{\infty} 2 A_{0} \cos \left(\tau+\phi_{0}\right) a_{2 n} \cos 2 n\left(\tau+\phi_{0}\right)\right. \\
& \left.-\sum_{n=0}^{\infty} 2 a_{2 n+1} \cos (2 n+1)\left(\tau+\phi_{0}\right)\right\}+\left(1+\frac{k}{\omega_{1}}\right) A_{0} \cos \left(\tau+\phi_{0}\right)+\frac{\mu \Omega}{\omega_{1}} A_{0} \sin \left(\tau+\phi_{0}\right) \\
= & \frac{F}{\omega_{1}} \cos \tau+\frac{\mu \Omega}{\omega_{1}} A_{0} \sin \left(\tau+\phi_{0}\right)+\frac{k}{\omega_{1}} \sum_{n=0}^{\infty}\left(2 a_{2 n+1}-A_{0} a_{2 n}-A_{0} a_{2 n+2}\right) \cos (2 n+1)\left(\tau+\phi_{0}\right) \\
& +\left(1+\frac{k}{\omega_{1}}\right) A_{0} \cos \left(\tau+\phi_{0}\right) .
\end{aligned}
$$

From Eq. (53), the necessary conditions of no secular term in $x_{1}$ are

$$
\begin{array}{r}
\left(\omega_{1}+k\right) A_{0}+2 k a_{1}-k A_{0}\left(a_{0}+a_{2}\right)+F \cos \phi_{0}=0, \\
\mu A_{0} \Omega+F \sin \phi_{0}=0 .
\end{array}
$$

When Eqs. (54) and (55) are satisfied, Eq. (53) reduces to

$$
x_{1}^{\prime \prime}+x_{1}=\frac{k}{\omega_{1}} \sum_{n=1}^{\infty}\left(2 a_{2 n+1}-A_{0} a_{2 n}-A_{0} a_{2 n+2}\right) \cos (2 n+1)\left(\tau+\phi_{0}\right) .
$$

The particular solution to Eq. (56) is

$$
x_{1}=\frac{k}{\omega_{1}} \sum_{n=1}^{\infty} \frac{2 a_{2 n+1}-A_{0} a_{2 n}-A_{0} a_{2 n+2}}{1-(2 n+1)^{2}} \cos (2 n+1)\left(\tau+\phi_{0}\right),
$$

where for any particular $\Omega, A_{0}$ and $\phi_{0}$ can be given by Eqs. (54) and (55). Thus, the first-order approximation of $x$ is

$$
\begin{aligned}
x(t)= & x_{0}(\Omega t)+\alpha x_{1}(\Omega t) \\
= & A_{0} \cos \left(\Omega t+\phi_{0}\right) \\
& +\frac{k \alpha}{\omega_{1}} \sum_{n=1}^{\infty} \frac{2 a_{2 n+1}-A_{0} a_{2 n}-A_{0} a_{2 n+2}}{1-(2 n+1)^{2}} \cos (2 n+1)\left(\Omega t+\phi_{0}\right),
\end{aligned}
$$

where $k=\frac{1-\bar{k}}{\varepsilon}, \alpha$ is given by Eq. (41), in which $\omega_{1} \approx \frac{\Omega^{2}-1}{\varepsilon}$ based on Eq. (40) for the first-order L-P approximation. And finally, one has

$$
\begin{aligned}
x(t)= & A_{0} \cos \left(\Omega t+\phi_{0}\right) \\
& +\frac{1-\bar{k}}{\Omega^{2}} \sum_{n=1}^{\infty} \frac{2 a_{2 n+1}-A_{0} a_{2 n}-A_{0} a_{2 n+2}}{1-(2 n+1)^{2}} \cos (2 n+1)\left(\Omega t+\phi_{0}\right) .
\end{aligned}
$$

Therefore, the modified L-P method gives an approximate periodic solution to Eq. (6) when $\bar{\mu}>0$ and $\bar{F}>0$, according to which the frequency-response curves are plotted and compared to those from numeric simulations in Fig. 17. 


\subsection{Comparison and Analysis}

It should be noted that for the results of the modified L-P method, a truncated version of Eq. (59) is used for plotting Fig. 17. Eq. (59) is truncated so that the discarded terms will not have much influence on the shape of limit cycles.

$$
\begin{aligned}
x \approx & A_{0} \cos \left(\Omega t+\phi_{0}\right) \\
& +\frac{1-\bar{k}}{\Omega^{2}} \sum_{n=1}^{7} \frac{2 a_{2 n+1}-A_{0} a_{2 n}-A_{0} a_{2 n+2}}{1-(2 n+1)^{2}} \cos (2 n+1)\left(\Omega t+\phi_{0}\right) .
\end{aligned}
$$

It also should be mentioned that all the parameters are held the same for both the modified L-P method and numerical simulation for each figure; so $\bar{\mu}=0.05$, and $\bar{F}=2.0$ for all the figures, while $\bar{k}$ varies.

As seen from Fig. 17, modified L-P results match satisfactorily within major part of the frequency range. Only for the small frequencies, frequency-response curves from the two methods differ.
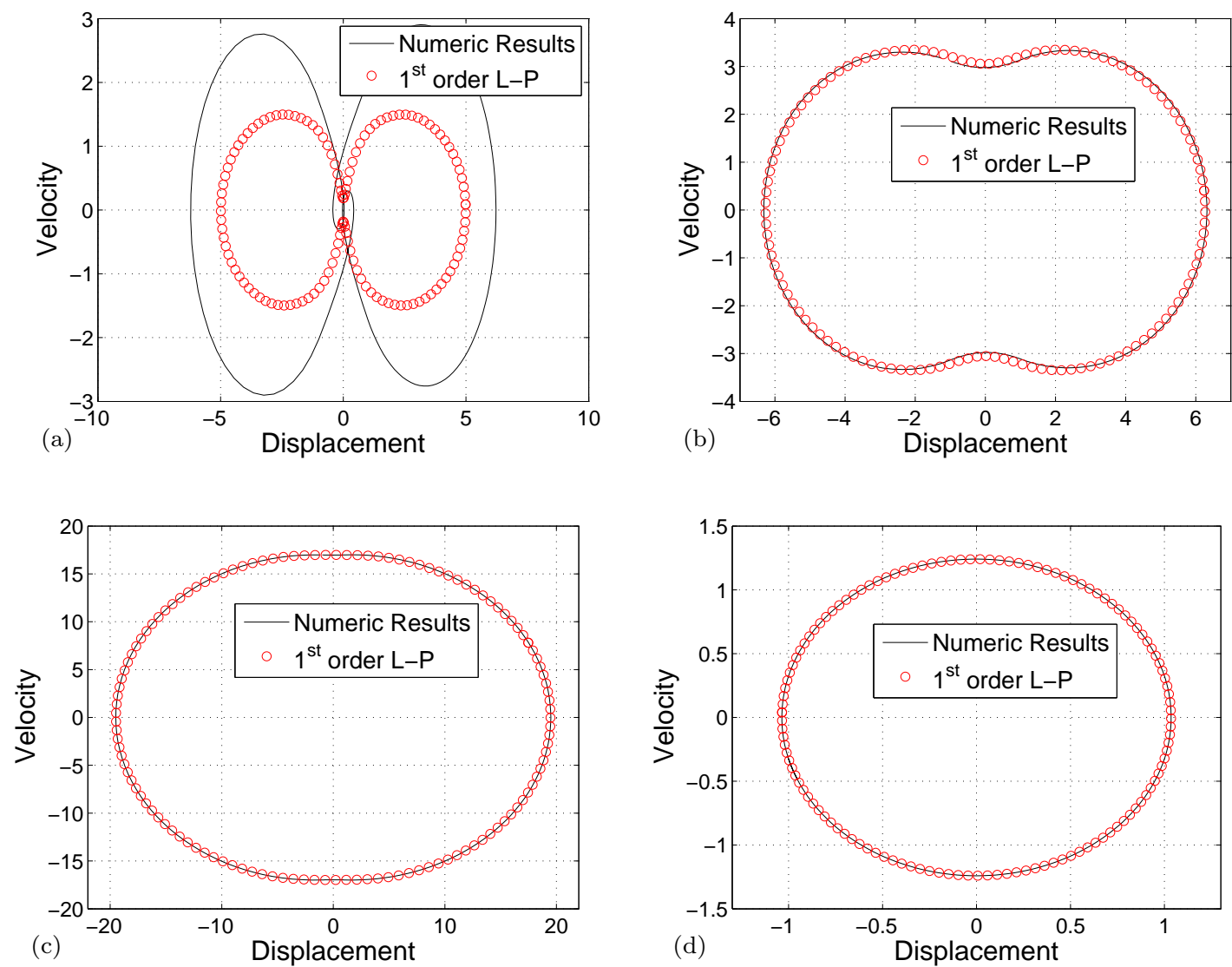

Fig. 18. Forced and damped limit cycles for $\bar{k}=-0.5, \bar{\mu}=0.05$, and $\bar{F}=2.0$. (a) $\Omega=0.30$; (b) $\Omega=0.60$; (c) $\Omega=0.90 ;$ (d) $\Omega=1.20$.

From Fig. 18, limit cycles from the modified L-P results agree satisfactorily with the numerical simulations for the higher frequencies. While for lower frequencies, they have quite a big difference. The major reason is that for lower frequencies, the actual limit cycles, although are still centrally symmetric, are not axially symmetric any more, but the first-order modified L-P approximation does not include any sine terms that would otherwise break the axial symmetry. Only approximations with order higher than first include sine 
terms. The amplitudes of the limit cycles in Fig. 18 are in agreement with the frequency response curve in Fig. 17(a).

It should be mentioned that all the numerical results in Figs. 9-18 are obtained through the algorithm based on a continuation method and an event-driven simulation technique.

\subsection{When $\bar{k}$ is Positive}

Although the analytical solution of piecewise linear dynamic system with negative stiffness is the primary motivation of the present work, it is worth mentioning that Eqs. (54)-(55) and (60) apply to piecewise linear systems with positive stiffnesses as well. In order to illustrate this, when $\bar{k}$ is positive, the frequency-response curves are plotted and compared in Fig. 19 to those from numeric simulations based on the continuation method and the event-driven simulation technique. All the parameters are held the same for both modified L-P method and numerical simulation for each figure. $\bar{F}=5.0$ and $\bar{\mu}=0.2$ for the figures in Fig. 19, while $\bar{k}$ varies.
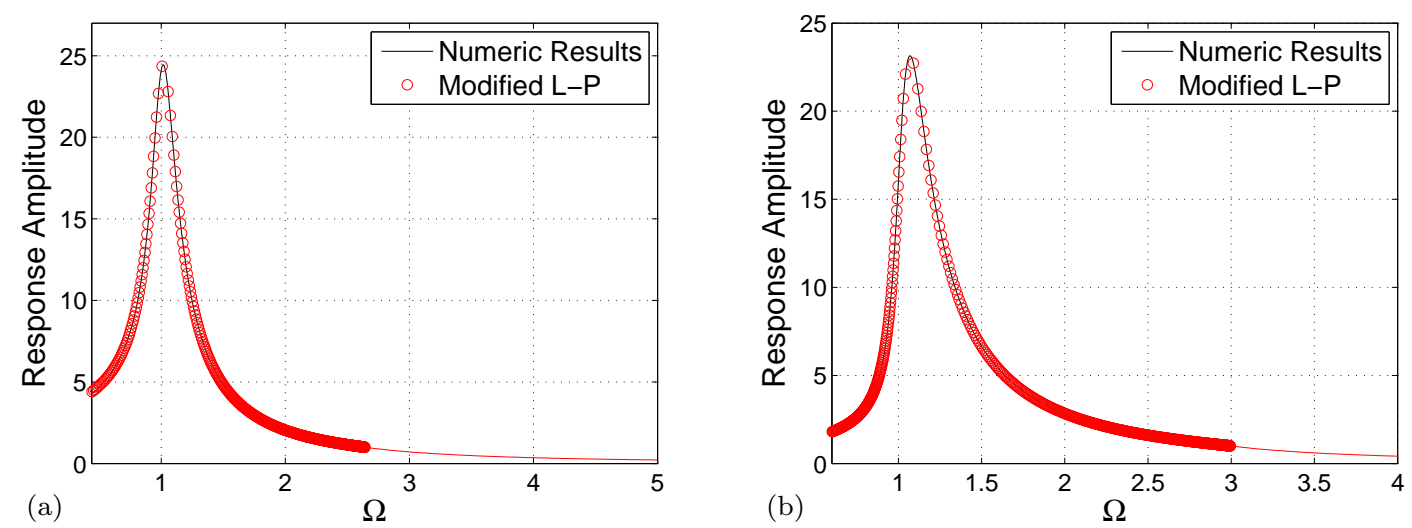

Fig. 19. Forced and damped frequency-response curves when $\bar{k}$ is positive. (a) $\bar{k}=2.0$; (b) $\bar{k}=4.0$.

As seen from Fig. 19, numerical results and modified L-P results match perfectly well with each other.

\section{CONCLUSIONS}

Physical insights into a new piecewise linear system with initial negative stiffness followed by positive stiffness has been studied and its advantages in practical dynamic system design have been presented.

The existence of the negative stiffness reduces the peak restoring force at the cost of increasing the peak displacement. A combination of negative stiffness and large damping is found to effectively reduce the peak restoring force with slightly increased peak displacement, which can be useful in a dynamic system in reducing the force transmitted. The time histories and limit cycles from numerical simulations that support these conclusions are presented. The oscillations are found to converge to limit cycles around the two equilibrium points on the two sides due to the double-well potential. Initial negative stiffness followed by positive stiffness in this piecewise linear system and the resultant double-well potential play a central role in ensuring that the oscillations reach a limit cycle eventually. In this sense, the dynamic behavior of this piecewise linear system resembles that of a van der Pol oscillator with negative damping followed by positive damping.

An exact solution for the free vibration of the non-dimensionalized piecewise linear system with initial negative stiffness followed by positive stiffness is derived. The influence of the middle segment is found not to be significant when the response amplitude is very large. Free vibration of the piecewise linear system asymptotically approaches that of the purely linear system with non-dimensionalized stiffness one as the displacement amplitude goes to infinity. An algorithm implemented specifically for simulating piecewise 
linear systems is adopted to obtain frequency-response curves. The evolution of the frequency-response curve is investigated by increasing the magnitude of the stiffness ratio $\bar{k}$ in the non-dimensionalized piecewise linear system. A detached loop is found to arise as the magnitude of the stiffness ratio become large. The modified L-P solution is derived for the forced and damped case, which is found to agree satisfactorily with the numeric simulations, and is quite accurate whether $\bar{k}$ is negative or positive.

With an appropriate choice of negative stiffness and large damping, the investigated piecewise linear system is useful for dynamic system design because the force transmitted can be reduced significantly. Different requirements of dynamic system design can be satisfied by choosing suitable combination of stiffness and damping.

The presented analysis is a special case study that requires further analytical work to make it more general, which is a subject of a future study.

\section{ACKNOWLEDGMENT}

The authors also wishes to gratefully acknowledge Dr. Andrew J. Dick for his suggestions. The work was carried out with the financial support of China Scholarship Council (CSC). Its sponsorship is gratefully acknowledged.

\section{References}

[1] Shaw SW, Holmes PJ. A periodically forced piecewise linear oscillator. Journal of Sound and Vibration 1983;90(1):129-155.

[2] Wiercigroch M, Sin VWT. Experimental study of a symmetrical piecewise base-excited oscillator. Journal of Applied Mechanics 1998;65:657-663.

[3] Luo ACJ. A theory for non-smooth dynamic systems on the connectable domains. Commun. Nonlinear Sci. Numer. Simulat. 2004;10(1):1-55.

[4] Luo ACJ. A periodically forced, piecewise linear system, Part I: Local singularity and grazing bifurcation. Commun. Nonlinear Sci. Numer. Simulat. 2004;12(3):379-396.

[5] Luo ACJ. A periodically forced, piecewise linear system, Part II: The fragmentation mechanism of strange attractors and grazing. Commun. Nonlinear Sci. Numer. Simulat. 2004;12(6):986-1004.

[6] Vestroni F, Luongo A, Paolone A. A perturbation method for evaluating nonlinear normal modes of a piecewise linear two-degree-of-freedom system. Nonlinear Dynamics 2008;54:379-393.

[7] Pilipchuk VN. Closed-form periodic solutions for piecewise-linear vibrating systems. Nonlinear Dynamics 2009;58:169-178.

[8] Di Bernardo M, Budd CJ, Champneys AR, Kowalczyk P. Piecewise-Smooth Dynamical Systems: Theory and Applications. New York, USA: Springer Verlag; 2008.

[9] Piiroinen P and Kuznetsov YA. An event-driven method to simulate filippov systems accurate computing of sliding motions. Technical Report, University of Bristol; 2005.

[10] Murty KG. Linear Complementarity, Linear and Nonlinear Programming, Sigma Series in Applied Mathematics. 3. Berlin, Germany: Heldermann Verlag; 1988

[11] Filippov A.Differential Equations with Discontinuous Right-Hand Sides, Mathematics and Its Applications (Soviet Series), 18. Dordrecht: Kluwer Academic Publishers Group; 1988.

[12] Nagarajaiah S, Reinhorn AM, Constantinou MC, Taylor DP, Pasala DTR, Sarlis AA. Adaptive negative stiffness: A new structural modification approach for seismic protection. 5th World Conference on Structural Control and Monitoring, Tokyo, Japan; 2010.

[13] Pasala DTR, Sarlis AA, Nagarajaiah S, Reinhorn AM, Constantinou MC, D. Taylor DP. Adaptive negative stiffness: A new structural modification approach for seismic protection. Journal of Structural Engineering 2012;139:1112-1123.

[14] Sarlis AA, Pasala DTR, Constantinou MC, Reinhorn AM, Nagarajaiah S, Taylor DP. Negative stiffness device for seismic protection of structures. Journal of Structural Engineering 2012;139:1124-1133.

[15] Pasala DTR, Sarlis AA, Reinhorn AM, Nagarajaiah S, Constantinou MC, Taylor D. Simulated bilinear-elastic behavior in a sdof elastic structure using negative stiffness device: Experimental and analytical study. Journal of Structural Engineering 2013;140(2):04013049. doi:10.1061/(ASCE)ST.1943-541X.0000830.

[16] Nagarajaiah S, Reinhorn A. Applicability of pseudo-force method to highly nonlinear dynamic problems. Analysis and Computation 1994, New York, NY, USA; 1994; pp. 165-172.

[17] Carrella A, Brennan MJ, Kovacic I, Waters TP. On the force transmissibility of a vibration isolator with quasi-zero-stiffness. Journal of Sound and Vibration 2009;322:707-717.

[18] Van der Pol B. On oscillation hysteresis in a simple triode generator. Philosophical Magazine 1922;43:700-719.

[19] Van Dyke M. Perturbation Methods in Fluid Mechanics. Stanford, California, USA: The Parabolic Press; 1975.

[20] Nayfeh AH. Introduction to Perturbation Techniques. New York, USA: Wiley-Interscience; 1993.

[21] Burton TD. A perturbation method for certain nonlinear oscillators. International Journal for Non-linear Mechanics 1982;19:397-407. 
[22] Burton TD, Rahman Z. On the multi-scale analysis of strongly non-linear forced oscillators. International Journal for Non-linear Mechanics 1986;21:135-146.

[23] Cheung YK, Chen SH, Lau SL. A modified Lindstedt-Poincaré method for certain strongly non-linear oscillators. International Journal for Non-linear Mechanics 1991;26:367-378.

[24] Luo ACJ., Albert CJ. Discrete and Switching Dynamical Systems, Monograph Series in Mathematical Methods and Modeling. Beijing/Dordrecht: Higher Education Press/L\& H Scientific; 2012.

[25] Luo ACJ., Albert CJ. Discontinuous Dynamical Systems on Time-varying Domains, Monograph Series in Nonlinear Physical Science. Beijing/Dordrecht: Higher Education Press/Springer; 2009.

[26] Hedlund S. Computational methods for hybrid systems. PhD Dissertation. Lund, Sweden: Lund Institute of Technology; 1999.

[27] Arango I, Taborda JA. Continuation of nonsmooth bifurcations in filippov systems using singular point tracking. International Journal of Applied Mathematics and Informations 2007;1(1):36-49.

[28] Pasala DTR. Control of structural systems using novel adaptive and adaptive-passive devices. PhD Dissertation. Houston: Rice University; 2012.

[29] Allgower EL, Georg K. Numerical Continuation Methods: An Introduction. New York, NY, USA: Springer-Verlag New York, Inc.; 1990. 